\title{
Rhinacanthin-C Mediated Herb-Drug Interactions with Drug Transporters and Phase I Drug-Metabolizing Enzymes
}

\author{
(1) Wilasinee Dunkoksung, (1) Nontima Vardhanabhuti, Pongpun Siripong, and (1) Suree Jianmongkol
}

Departments of Pharmacology and Physiology (W.D., S.J.) and Pharmaceutics and Industrial Pharmacy (N.V.), Faculty of Pharmaceutical Sciences, Chulalongkorn University, Bangkok, Thailand; and National Cancer Institute, Bangkok, Thailand (P.S.)

Received November 26, 2018; accepted July 15, 2019

\begin{abstract}
Rhinacanthin-C is a major active constituent in Rhinacanthus nasutus (L.) Kurz, a plant widely used in herbal remedies. Its potential for pharmacokinetic herb-drug interaction may exist with drug transporters and drug metabolizing enzymes. This study assessed the possibility for rhinacanthin-C-mediated drug interaction by determining its inhibitory effects against major human efflux and influx drug transporters as well as various human cytochrome P450(CYP) isoforms. Rhinacanthin-C demonstrated a moderate permeability through the Caco-2 monolayers $\left[P_{\text {app (AP-to-BL) }}=1.26 \times\right.$ $10^{-6} \mathrm{~cm} / \mathrm{s}$ ]. It significantly inhibited transport mediated by both

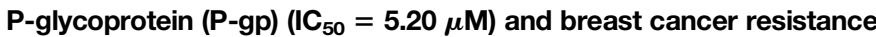
protein (BCRP) $\left(\mathrm{IC}_{50}=0.83 \mu \mathrm{M}\right)$ across Caco-2 and BCRPoverexpressing Madin-Darby canine kidney II cells (MDCKII) cells. This compound also strongly inhibited uptake mediated by organic
\end{abstract}

anion-transporting polypeptide 1B1 (OATP1B1) $\left(\mathrm{IC}_{50}=0.70 \mu \mathrm{M}\right)$ and OATP1B3 $\left(\mathrm{IC}_{50}=3.95 \mu \mathrm{M}\right)$ in OATP1B-overexpressing HEK cells. In addition to its inhibitory effect on these drug transporters, rhinacanthin-C significantly inhibited multiple human CYP isoforms including CYP2C8 $\left(\mathrm{IC}_{50}=4.56 \mu \mathrm{M}\right), 2 \mathrm{C} 9\left(\mathrm{IC}_{50}=1.52 \mu \mathrm{M}\right), 2 \mathrm{C} 19\left(\mathrm{IC}_{50}=\right.$ $28.40 \mu \mathrm{M})$, and $3 \mathrm{~A} 4 / 5\left(\mathrm{IC}_{50}=53 \mu \mathrm{M}\right.$ for midazolam and $\mathrm{IC}_{50}=$ 81.20 $\mu \mathrm{M}$ for testosterone), but not CYP1A2, 2A6, 2B6, 2D6, and 2E1. These results strongly support a high propensity for rhinacanthin-C as a perpetrator of clinical herb-drug interaction via inhibiting various influx and efflux drug transporters (i.e., P-gp, BCRP, OATP1B1, and OATP1B3) and CYP isoforms (i.e., CYP2C8, CYP2C9, and CYP2C19). Thus, the potential for significant pharmacokinetic herb-drug interaction should be addressed when herbal products containing rhinacanthin-C are to be used in conjunction with other prescription drugs.

\section{Introduction}

Herbal products have been used increasingly worldwide either as alternative medicines or dietary supplements. Coadministration of these products with therapeutic agents potentially leads to herb-drug interaction via pharmacokinetic interference on drug metabolism and/or transport (Oga et al., 2016; Sprouse and van Breemen, 2016; Wu et al., 2016). The common interference mechanisms involve inhibition and induction of drug metabolizing enzymes and drug transporters. Consequently, both therapeutic efficacy and safety can be affected (Zhou et al., 2007; Oga et al., 2016).

The superfamily of cytochrome P450 (CYP) enzymes-particularly CYP1A2, CYP2B6, CYP2C8, CYP2C9, CYP2C19, CYP2D6, and CYP3A4/5-represents the major drug-metabolizing enzymes in phase I oxidative metabolism. The metabolism of approximately $70 \%$ of drugs and exogenous substances in humans is attributed to these expressed CYP isoforms in the liver and extrahepatic tissues (i.e., intestines) (Wienkers and Heath, 2005). When taken orally, CYP substrates are

This work was supported by the 100th Anniversary Chulalongkorn University Fund for Doctoral Scholarship and the 90th Anniversary of Chulalongkorn University Fund (Ratchadaphiseksomphot Endowment Fund).

https://doi.org/10.1124/dmd.118.085647 metabolized by intestinal CYP enzymes as well as hepatic CYP enzymes. Both intestinal and hepatic metabolism will affect drug absorption and disposition, resulting in decreased bioavailability and altered pharmacokinetic profiles of those substrate drugs (Wienkers and Heath, 2005; Xie et al., 2016).

Moreover, drugs that are subjected to CYP enzyme metabolism are at high risk for drug-drug interactions (DDIs) when orally coadministered with CYP inhibitors. If CYP inhibitors are present in the gastrointestinal (GI) tract at high concentrations, they can effectively inhibit the intestinal CYP-mediated metabolism of concomitant substrate drugs. In addition, if these inhibitors reach the liver at high levels, they can also interfere with hepatic drug metabolism, leading to even higher plasma drug concentrations and alteration in therapeutic responses. Drugs with narrow therapeutic index such as phenytoin and warfarin are more vulnerable to such DDIs (Miners and Birkett, 1998).

Efflux transporters (e.g., breast cancer resistance protein [BCRP], multidrug resistance-associated protein [MRP], P-glycoprotein [P-gp]) and influx transporters (e.g., organic anion-transporting polypeptide [OATP]) also play important roles in drug absorption and disposition (Mizuno et al., 2003; König et al., 2013). These transporters are located in various organs, including the intestine, liver, and kidney. Several popularly used herbal products such as St. John's wort, echinacea, goldenseal, grapefruit juice, ginseng, and milk thistle are potent inhibitors

ABBREVIATIONS: ACN, acetonitrile; AP-to-BL, apical-to-basolateral; BCRP, breast cancer resistance protein; BL-to-AP, basolateral-to-apical; Caco-2, human colon adenocarcinoma cells; CYP, cytochrome P450 isoform; DDI, drug-drug interaction; 8-FcA, 8-fluorescein-cAMP; G418, geneticin; GI, gastrointestinal; HBSS, Hanks' balanced salt solution; HEK293, human embryonic kidney 293 cells; HLMs, human liver microsomes; Ko143, tert-butyl 3-[(2S,5S,8S)-14-methoxy-2-(2-methylpropyl)-4,7-dioxo-3,6,17-triazatetracyclo[8.7.0.0 $0^{3,8} \cdot 0^{11,16}$ ]heptadeca-1(10),11,13,15-tetraen-5-yl] propanoate; MDCKII, Madin-Darby canine kidney II cells; MEM, minimal essential medium; MRP, multidrug resistance-associated protein; OATP, organic anion-transporting polypeptide; $\mathrm{P}_{\mathrm{app}}$, apparent permeability coefficient; P-gp, P-glycoprotein; PSC 833, valspodar; TEER, transepithelial electrical resistance; UHPLC-MS/MS, ultrahigh-pressure liquid chromatography with tandem mass spectrometry. 
or modulators of various CYP enzymes and transporter proteins (Gurley et al., 2005; Brantley et al., 2014). Clinically, the incidence of CYP-/ transporter-based drug interactions from herb-mediated pharmacokinetic alteration of prescription medicines has been reported (Oga et al., 2016; Sprouse and van Breemen, 2016). Hence, investigation of potential herb-drug interaction relating to CYP enzymes and transporters is necessary to support efficacy and safety of therapeutic agents in concurrent use with herbal products.

Rhinacanthus nasutus (L.) Kurz (Acanthaceae) has long been used in traditional medicines in the tropical region including India, Taiwan, Thailand, and the south of the People's Republic of China. The plant has been used to treat various symptoms such as fever, fluid retention, hypertension, pneumonia, hepatitis, diabetes, and cancers (Siripong et al., 2006a,b; Horii et al., 2013). Rhinacanthin-C (Fig. 1) is a major bioactive naphthoquinone constituent found in this plant (Sendl et al., 1996; Siripong et al., 2006a,b; Panichayupakaranant et al., 2009). Recently, we demonstrated that rhinacanthin-C significantly enhanced doxorubicin-mediated cytotoxicity in vitro via inhibition of MRP2 and P-gp efflux transporters (Wongwanakul et al., 2013; Chaisit et al., 2017). In addition to its effect on efflux transporters, this naphthoquinone compound exerts inhibitory effect on a few CYP enzymes (i.e., CYP2A6 and 2A13) (Pouyfung et al., 2014). Thus, it can be anticipated that rhinacanthin- $\mathrm{C}$ may cause herb-drug pharmacokinetic interaction when concurrently used with CYP/transporter drug substrates. Until now, there have been no reports on rhinacanthin-C inhibition against P-gp, BCRP, or OATP drug transporters or against human CYP enzymes, particularly those involved in drug metabolism.

This study assesses the potential for herb-drug interaction of rhinacanthin- $\mathrm{C}$ when taken orally. We examined the intrinsic properties of this compound as a perpetrator to interfere with drug absorption and disposition via drug transporters and phase I drug-metabolizing enzymes. The results of this mechanistic study on drug interaction were also used as a basis in predicting the potential for clinical pharmacokinetic interference between rhinacanthin-C and other coadministered drugs.

\section{Materials and Methods}

Materials and Chemicals. Rhinacanthin-C was isolated from the root of Rhinacanthus nasutus (L.) Kurz (R. nasutus), purified, and identified as previously described elsewhere (Siripong et al., 2006a,b). Other chemicals, including acyclovir, amodiaquin dihydrochloride dihydrate, atenolol, dextromethorphan hydrobromide, tert-butyl 3-[(2S,5S,8S)-14-methoxy-2-(2-methylpropyl)-4,7dioxo-3,6,17-triazatetracyclo[8.7.0.0 $\left.0^{3,8} \cdot 0^{11,16}\right]$ heptadeca-1(10),11,13,15-tetraen-5-yl] propanoate (Ko143), propranolol, tolbutamide, valspodar, and Hanks' balanced salt solution (HBSS) were purchased from Sigma-Aldrich (St. Louis, MO). Bupropion hydrochloride, chlorzoxazone, coumarin, digoxin, digitoxin, doxazosin mesylate, ketoconazole, $\beta$-nicotinamide adenine dinucleotide phosphate (NADPH), orphenadrine hydrochloride, phenacetin, prazosin hydrochloride, and testosterone were purchased from Tokyo Chemical Industry (Tokyo, Japan). We obtained 8-fluorescein-cAMP (8-FcA) from Biolog Life Science Institute (Bremen, Germany), and (S)-mephenytoin from Cayman Chemical (Ann Arbor, MI). Midazolam was purchased from Cerilliant Corporation (Round Rock, TX). Dulbecco's modified Eagle's medium (CYP enzymes), geneticin (G418),

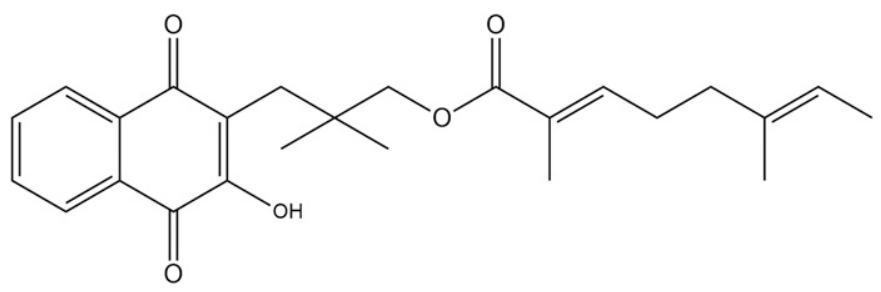

Fig. 1. Chemical structure of rhinacanthin-C.
GlutaMax, L-glutamine, and minimal essential medium (MEM) were from Gibco/ Life Technologies (Grand Island, NY). Fetal bovine serum (FBS) was from Biochrom AG (Berlin, Germany). Hygromycin and pooled human liver microsomes (HLMs) from 50 donors were obtained from Invitrogen (Carlsbad, CA). All other reagents were of high-performance liquid chromatography or analytic grade. Transwells ( $12 \mathrm{~mm}$ diameter, $0.4 \mu \mathrm{m}$ pores) and 24 -well plates coated with $0.1 \mathrm{mg} / \mathrm{ml}$ poly-D-lysine were purchased from Corning (Corning, NY).

Cell Cultures. The human colon adenocarcinoma (Caco-2, HTB37) cell line was obtained from the American Type Culture Collection (ATCC, Manassas, VA). The cells were cultured in CYP enzymes containing $10 \%$ FBS, $1 \%$ nonessential amino acids, $1 \% \mathrm{~L}$-glutamine, and $1 \%$ penicillin/streptomycin solution at $37^{\circ} \mathrm{C}$ in a humidified atmosphere of $5 \% \mathrm{CO}_{2}$. For the transport assays, cells (passage numbers 40-60) were seeded at a density of $6.0 \times 10^{4}$ cells $/ \mathrm{cm}^{2}$ onto Transwell inserts and cultured for 21 days. The integrity of cell monolayers was evaluated by measuring the transepithelial electrical resistance (TEER) with a Millicell-ERS (Millipore, Bedford, MA). Only Caco-2 monolayers having TEER values above $600 \Omega \mathrm{cm}^{2}$ were used in our experiments.

The polarized Madin-Darby canine kidney II (MDCKII) parental cell line and subclone transduced with human BCRP (MDCKII-BCRP) were kind gifts from Dr. Alfred H. Schinkel (Netherlands Cancer Institute, Amsterdam, the Netherlands). The cells were maintained in CYP enzymes containing 10\% FBS, $1 \%$ GlutaMax, and $0.5 \%$ penicillin/streptomycin solution in a humidified atmosphere of $5 \% \mathrm{CO}_{2}$ at $37^{\circ} \mathrm{C}$. For transport assays, cells were seeded at a density of $21.4 \times 10^{4}$ cells $/ \mathrm{cm}^{2}$ onto Transwell inserts and grown for 3 days. The cell monolayers with TEER values above $200 \Omega \mathrm{cm}^{2}$ were used in our experiments.

The human embryonic kidney 293 (HEK293) cells stably overexpressing human OATP1B1 (HEK-OATP1B1), human OATP1B3 (HEK-OATP1B3), and the vector control cell lines HEKCo/G418 and HEK-Co/Hygromycin were kind gifts from Dr. Jörg König (Institute of Experimental and Clinical Pharmacology and Toxicology, Friedrich-Alexander-Universität Erlangen-Nürnberg, Erlangen, Germany). The cells were cultured in 10\% FBS-MEM containing either geneticin or hygromycin at $37^{\circ} \mathrm{C}$, as previously described elsewhere (König et al., 2011, 2012). For uptake assays, cells were seeded at densities of $12.5 \times 10^{4} \mathrm{cells} / \mathrm{cm}^{2}$ (for HEK-OATP1B1 and HEKCo/G418) and $8.0 \times 10^{4} \mathrm{cells} / \mathrm{cm}^{2}$ (for HEKOATP1B3 and HEK-Co/Hygromycin) onto poly-D-lysine coated plates and grown to their confluence for 3 days. The cells were further cultured in the presence of $10 \mathrm{mM}$ sodium butyrate for 1 day and used for the uptake experiment (König et al., 2011)

Permeability Assays. Permeability assays were performed as previously described elsewhere (Hubatsch et al., 2007; Dunkoksung et al., 2019). The Caco-2 monolayers were treated with $10 \mathrm{mM}$ HEPES-HBSS ( $\mathrm{pH}$ 7.4) containing either rhinacanthin-C or a cocktail mixture of three permeability markers $(10 \mu \mathrm{M}$ acyclovir, $10 \mu \mathrm{M}$ atenolol, and $10 \mu \mathrm{M}$ propranolol) in the apical (AP) site at $37^{\circ} \mathrm{C}$ for 3 hours. Samples were collected from the basolateral (BL) side every 30 minutes with fresh buffer replacement. The collected samples were mixed with an equal volume of $100 \%$ acetonitrile $(\mathrm{ACN})$ containing the internal standard (75 nM labetalol for permeability markers; $1.25 \mu \mathrm{M}$ menadione sodium bisulfate for rhinacanthin-C). After centrifugation $\left(12,000 \mathrm{~g}\right.$ for 10 minutes at $\left.4^{\circ} \mathrm{C}\right)$, the supernatants were analyzed by ultrahigh-pressure liquid chromatography with tandem mass spectrometry (UHPLC-MS/MS).

P-gp and BCRP Substrate Assays. The bidirectional transports (AP to BL and $\mathrm{BL}$ to $\mathrm{AP}$ directions) of rhinacanthin-C were determined across cell monolayers (Caco-2 monolayers for P-gp; MDCKII-BCRP/MDCKII-parental monolayers for BCRP) at $37^{\circ} \mathrm{C}$, using a protocol described elsewhere (Hubatsch et al., 2007; Poller et al., 2011; Dunkoksung et al., 2019). Rhinacanthin-C was added to either the AP or the BL chamber, depending on the transport direction studied. A known substrate of each transporter (P-gp substrate digoxin, $5 \mu \mathrm{M}$; BCRP substrate prazosin, $5 \mu \mathrm{M}$ ) was used as a positive control group. Samples were taken from the relevant chamber every 30 minutes for 180 minutes and were mixed with an equal volume of $100 \%$ ACN containing internal standard of each compound ( $2 \mu \mathrm{M}$ digitoxin for digoxin, $60 \mathrm{nM}$ doxazosin for prazosin, and $1.25 \mu \mathrm{M}$ menadione sodium bisulfate for rhinacanthin-C). After centrifugation, the supernatants were analyzed by UHPLC-MS/MS.

P-gp and BCRP Inhibition Assays. The inhibitory action of rhinacanthin-C on either P-gp or BCRP activity was determined in the bidirectional transport assays, as described earlier. The cell monolayers were treated with probe P-gp or BCRP substrates (digoxin, $5 \mu \mathrm{M}$; prazosin, $5 \mu \mathrm{M}$ ) in the presence or absence of rhinacanthin-C. Samples were collected from the relevant chamber every 
TABLE 1

Concentration and mass transition of the probe substrates, permeability markers, rhinacanthin-C, and internal standards

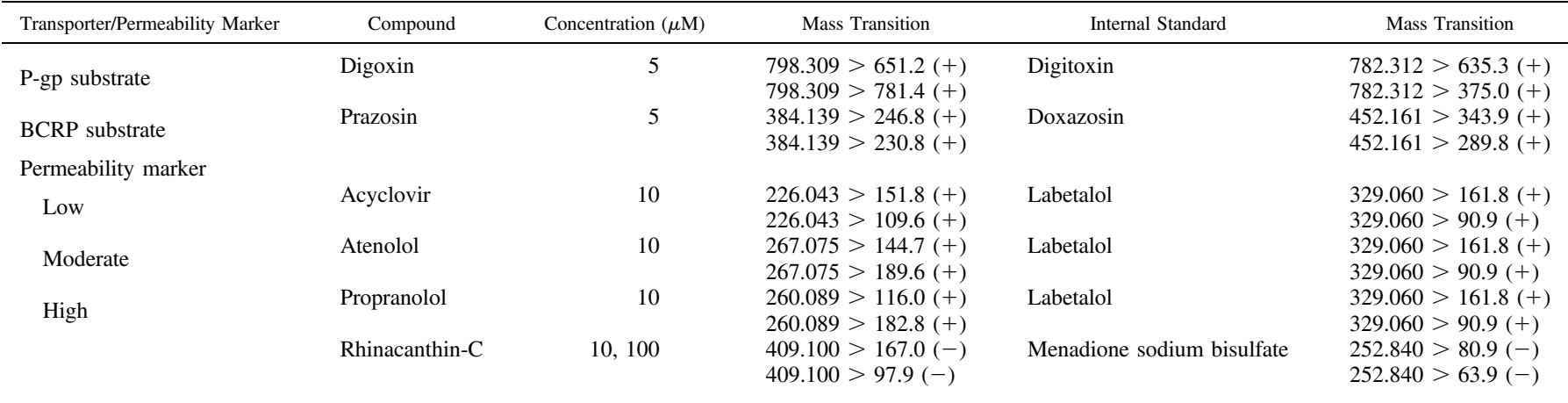

30 minutes for 180 minutes and were mixed with an equal volume of $100 \% \mathrm{ACN}$ containing the internal standard. After centrifugation, the supernatants were quantified for digoxin and prazosin by UHPLC-MS/MS analysis. Known inhibitors of each transporter - valspodar and ketoconazole (P-gp inhibitors) or Ko143 (BCRP inhibitor)—were used as positive control groups.

OATP1B1 and OATP1B3 Inhibition Assays. The inhibitory action of rhinacanthin-C on either OATP1B1 or OATP1B3 activities was determined in the uptake assay with a protocol described elsewhere (Seithel et al., 2007; Bednarczyk, 2010). The cells (HEK293-OATP1B1, HEK293-OATP1B3, and respective vector control cell lines) were treated with rhinacanthin-C for 30 minutes at $37^{\circ} \mathrm{C}$, followed by the addition of the probe substrate $8-\mathrm{FcA}$ at a concentration of $2.5 \mu \mathrm{M}$ for OATP1B1 or $1.25 \mu \mathrm{M}$ for OATP1B3 for another 10 minutes. The treated cells were washed with ice-cold HBSS and lysed with methanol/10 mM Tris solution (1:1) containing $1 \mathrm{mM}$ EDTA. The fluorescent intensity of 8-FcA was determined with a microplate reader (Wallac 1420 VICTOR 3; PerkinElmer, Waltham, MA) at 485/535 nm (excitation/emission wavelengths). Cyclosporin A and rifampicin (known OATP1B1/OATP1B3 inhibitors) were used as positive control groups.

CYP Cocktail Inhibition Assays. The inhibitory action of rhinacanthin-C on CYP enzymes in HLMs was determined as described elsewhere (Li et al., 2015). HLMs $(0.2 \mathrm{mg} / \mathrm{ml}$, final concentration) were incubated with a cocktail of CYP substrates and rhinacanthin- $\mathrm{C}$ in $0.1 \mathrm{M}$ phosphate buffer ( $\mathrm{pH} 7.4)$ containing $3.3 \mathrm{mM} \mathrm{MgCl}{ }_{2}$ at $37^{\circ} \mathrm{C}$. The concentration of HLMs was kept at $0.2 \mathrm{mg} / \mathrm{ml}$ to minimize nonspecific binding to HLMs (Obach, 2008). The cocktail preparation of CYP substrates contained $100 \mu \mathrm{M}$ phenacetin (CYP1A2), $1.5 \mu \mathrm{M}$ coumarin (CYP2A6), $12 \mu \mathrm{M}$ bupropion (CYP2B6), $1 \mu \mathrm{M}$ amodiaquine (CYP2C8), $100 \mu \mathrm{M}$ tolbutamide (CYP2C9), $50 \mu \mathrm{M}(S)$-mephenytoin (CYP2C19), $2.5 \mu \mathrm{M}$ dextromethorphan (CYP2D6), $15 \mu \mathrm{M}$ chlorzoxazone (CYP2E1), $2.5 \mu \mathrm{M}$ midazolam, and $50 \mu \mathrm{M}$ testosterone (CYP3A4/5). The reaction was initiated by the addition of NADPH (1.3 mM, final concentration). At the end of the 10-minute incubation period, the reaction was stopped with ice-cold 3\% formic acid in $5 \%$ ACN solution containing $0.1 \mu \mathrm{M}$ orphenadrine as internal standard. The samples were collected and centrifuged before UHPLC-MS/MS analysis.
Mechanism-Based CYP Inhibition Assays. Mechanism-based inhibition of CYP enzymes was assessed by $\mathrm{IC}_{50}$ shift method (de Ron and Rajaraman, 2012; Haque et al., 2017). HLMs (0.2 $\mathrm{mg} / \mathrm{ml}$, final concentration) were incubated for 30 minutes with rhinacanthin- $\mathrm{C}$ in $0.1 \mathrm{M}$ phosphate buffer ( $\mathrm{pH}$ 7.4) containing $3.3 \mathrm{mM} \mathrm{MgCl} 2$ at $37^{\circ} \mathrm{C}$ in the presence or absence of $1.3 \mathrm{mM} \mathrm{NADPH}$. The reaction was initiated by addition of a cocktail of CYP2C8, 2C9, 2C19, and 3A4/5 substrates. At the end of the10-minute incubation period, the reaction was terminated with ice-cold $3 \%$ formic acid in $5 \% \mathrm{ACN}$ solution containing $0.1 \mu \mathrm{M}$ orphenadrine as internal standard. The samples were collected and centrifuged before UHPLC-MS/MS analysis.

UHPLC-MS/MS Analysis. UHPLC-MS/MS analysis was conducted on a Eksigent Ekspert ultra LC 100 with QTRAP 6500 system (AB Sciex, Framingham, MA). A rapid UHPLC gradient with an ACE C18 column ( $3 \mu \mathrm{m}, 50 \times 1.0 \mathrm{~mm}$ i.d.) was used to perform a quick reverse-phase separation (10\%-95\% ACN with $0.1 \%$ formic acid for all metabolites, rhinacanthin-C and prazosin; 20\%-95\% ACN with $2 \mathrm{mM}$ ammonium formate for digoxin). The flow rate was set at $200 \mu \mathrm{l} /$ minute, and the column oven temperature was set at $45^{\circ} \mathrm{C}$. The injection volume was either $5 \mu \mathrm{l}$ (prazosin) or $10 \mu \mathrm{l}$ (all metabolites, rhinacanthin-C, and digoxin). Detection was performed using electrospray ionization with polarity switching, collision-induced dissociation, and selected reaction monitoring. The mass transitions of the metabolites, digoxin (ammonium adduct), prazosin, rhinacanthin- $\mathrm{C}$, and internal standards are listed in Tables 1 and 2 .

Calculation. For the transport assays, the apparent permeability coefficient, $\mathrm{P}_{\text {app }}$ (centimeter per second), for both AP to BL and BL to AP directions of each test compound was calculated from the following equation:

$$
\mathrm{P}_{\text {app }}=(\mathrm{dQ} / \mathrm{dt}) \times\left(1 / \mathrm{C}_{0} \mathrm{~A}\right)
$$

where $\mathrm{dQ} / \mathrm{dt}$ is the cumulative transport rate (nanomoles per minute), $\mathrm{C}_{0}$ is the initial drug concentration on the drug-introducing side (micromolar), and $\mathrm{A}$ is the surface area of the inserts $\left(1.12 \mathrm{~cm}^{2}\right.$ in 12 -wells). The efflux ratio was the ratio of $\mathrm{P}_{\text {app(BL-to-AP) }}$ to $\mathrm{P}_{\text {app(AP-to-BL). }}$.

TABLE 2

$K_{\mathrm{m}}$ values, concentration of cytochrome $\mathrm{P} 450$-specific probe substrates, and mass transition for metabolites and internal standard

\begin{tabular}{llcclc}
\hline Enzyme & \multicolumn{1}{c}{ Substrate } & \multicolumn{1}{c}{$K_{\mathrm{m}}(\mu \mathrm{M})^{a}$} & Concentration $(\mu \mathrm{M})$ & \multicolumn{1}{c}{ Metabolite } & Mass Transition \\
\hline CYP1A2 & Phenacetin & $112.7 \pm 10.9$ & 100 & Acetaminophen & $152.0>109.7(+)$ \\
CYP2A6 & Coumarin & $1.5 \pm 0.2$ & 1.5 & 7-Hydroxycoumarin & $161.1>133.2(-)$ \\
CYP2B6 & Bupropion & $125.2 \pm 14.0$ & 12 & Hydroxybupropion & $256.1>138.8(+)$ \\
CYP2C8 & Amodiaquine & $1.0 \pm 0.1$ & 1 & N-desethylamodiaquine & $328.1>282.9(+)$ \\
CYP2C9 & Tolbutamide & $110.7 \pm 11.6$ & 100 & Hydroxytolbutamide & $285.1>186.2(-)$ \\
CYP2C19 & (S)-mephenytoin & $52.5 \pm 10.6$ & 50 & ( \pm )-4'Hydroxymephenytoin & $235.0>132.6(+)$ \\
CYP2D6 & Dextromethorphan & $2.9 \pm 0.5$ & 2.5 & Dextrophan & $258.1>157.2(+)$ \\
CYP2E1 & Chlorzoxazone & $149.8 \pm 12.6$ & 15 & 6-Hydroxychlorzoxazone & $186.0>119.7(-)$ \\
CYP3A4/5 & Midazolam & $2.7 \pm 0.1$ & 2.5 & 1'-Hydroxymidazolam & $342.0>324.0(+)$ \\
& Testosterone & $50.5 \pm 5.6$ & 50 & 6 -Hydroxytestosterone & $304.8>268.9(+)$ \\
& Orphenadrine (internal standard) & & & $270.8>180.9(+)$ \\
\hline
\end{tabular}

${ }^{a}$ Li et al. (2015). 


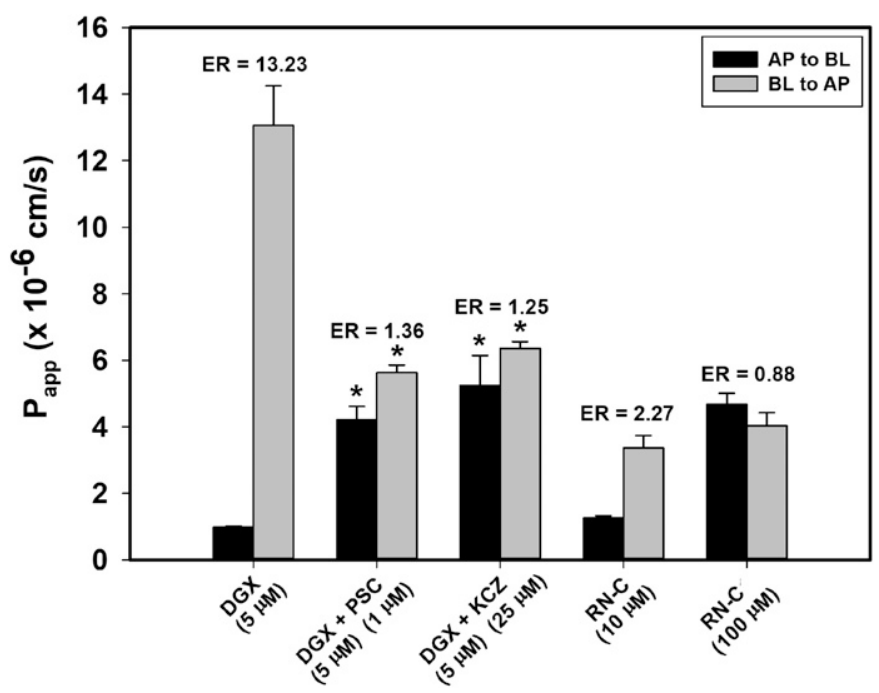

Fig. 2. Transport of rhinacanthin-C (RN-C) across the Caco- 2 monolayers from the apical-to-basolateral (AP-to-BL) direction and the basolateral-to-apical (BL-to-AP) direction. Digoxin (DGX) was used as a positive control substrate of P-gp. Valspodar (PSC 833; PSC) and ketoconazole (KCZ) were used as a positive control inhibitor of P-gp. Apparent permeability coefficients $\left(\mathrm{P}_{\mathrm{app}}\right)$ and efflux ratio (ER) represent the mean \pm S.E. of three independent experiments. ${ }^{*} P<0.05$ vs. control

Data Analysis. Quantitative UHPLC-MS/MS data were analyzed using MultiQuant Software (AB Sciex). The 50\% inhibitory concentration $\left(\mathrm{IC}_{50}\right)$ values were calculated from nonlinear regression analysis using SigmaPlot version 14.0 (Systat Software, San Jose, CA). Data were presented as mean \pm S.E. Data were obtained from three or four separated experiments. Statistical analysis was performed with either Student's $t$ test or ANOVA, followed by the post hoc Tamhane's T2 test. $P<0.05$ was considered statistically significant.

\section{Results}

Permeability of Rhinacanthin-C Across Caco-2 Cell Monolayer. Three permeability markers - namely acyclovir, atenolol, and propranolol (low, moderate, and high permeability, respectively)—were chosen as our references, based on the U.S. Food and Drug Administration (FDA) recommendation (CDER, 2017b). The $\mathrm{P}_{\text {app }}$ values across Caco-2 cell monolayers in the absorptive direction (AP-to-BL) of the three reference markers in ascending order were $0.06 \pm 0.01 \times 10^{-6} \mathrm{~cm} / \mathrm{s}$ (acyclovir, $10 \mu \mathrm{M}$ ), $0.48 \pm 0.06 \times 10^{-6} \mathrm{~cm} / \mathrm{s}$ (atenolol, $10 \mu \mathrm{M}$ ), and $19.25 \pm 0.99 \times 10^{-6} \mathrm{~cm} / \mathrm{s}$ (propranolol, $10 \mu \mathrm{M}$ ). The $\mathrm{P}_{\text {app (AP-to-BL) }}$ of rhinacanthin-C $(10 \mu \mathrm{M})$ was $1.26 \pm 0.07 \times 10^{-6} \mathrm{~cm} / \mathrm{s}$, suggesting that its permeability was likely in the same rank order with atenolol (moderately permeable compound).

P-gp and BCRP Substrate Assays. Expression of either P-gp in Caco-2 monolayers or BCRP in MDCKII-BCRP monolayers was clearly demonstrated by the bidirectional transport of specific probe substrates (digoxin, P-gp substrate; prazosin, BCRP substrate) and inhibitors (valspodar, ketoconazole, P-gp inhibitors; Ko143, BCRP inhibitor) (Figs. 2 and 3). The efflux ratio of digoxin $(5 \mu \mathrm{M})$ across Caco-2 monolayers significantly decreased by approximately 10 -fold in the presence of the positive control P-gp inhibitors (valspodar, $1 \mu \mathrm{M}$; ketoconazole, $25 \mu \mathrm{M}$ ) (Fig. 2). The known BCRP inhibitor Ko143 $(1 \mu \mathrm{M})$ significantly hindered permeation of prazosin $(5 \mu \mathrm{M})$ across MDCKII-BCRP monolayers by approximately 4-fold) but not in the MDCKII-control cell monolayers (Fig. 3).

Permeability of rhinacanthin- $\mathrm{C}$ in the absorptive direction $\left(\mathrm{P}_{\text {app }}\right.$ (AP-to-BL) across Caco-2 monolayers increased without any significant change in $\mathrm{P}_{\text {app (BL-to- AP) }}$ upon increasing its concentration from 10 to $100 \mu \mathrm{M}$ (Fig. 2). Consequently, the calculated efflux ratio of this compound somewhat decreased from $2.27 \pm 0.28(10 \mu \mathrm{M})$ to $0.88 \pm 0.09$ $(100 \mu \mathrm{M})$. This result suggested that rhinacanthin-C could be a weak P-gp substrate. On the other hand, the permeability profiles of rhinacanthin-C $(10,100 \mu \mathrm{M})$ across MDCKII-BCRP and MDCKIIcontrol monolayers were comparable, with the efflux ratio values of less than 2, suggesting that rhinacanthin-C is not a substrate for BCRP (Fig. 3).

P-gp and BCRP Inhibition by Rhinacanthin-C. The abilities of rhinacanthin-C to inhibit P-gp and BCRP activities were assessed by determining the net flux of digoxin and prazosin across cell monolayers. Rhinacanthin-C was able to inhibit both P-gp-mediated transport of digoxin and BCRP-mediated transport of prazosin in a concentrationdependent manner, with $\mathrm{IC}_{50}$ values of $5.20 \pm 0.44$ and $0.83 \pm 0.09 \mu \mathrm{M}$, respectively (Fig. 4; Table 3 ). Our results suggested that rhinacanthin-C was approximately 6-fold more selective for BCRP than for P-gp. At $10 \mu \mathrm{M}$, rhinacanthin- $\mathrm{C}$ inhibited transport of digoxin across Caco-2 monolayers by $64 \%$, and transport of prazosin across MDCKII-BCRP monolayers by $75 \%$.

OATP1B1 and OATP1B3 Inhibition by Rhinacanthin-C. Inhibition of OATP1B1 and OATP1B3 by rhinacanthin-C was investigated
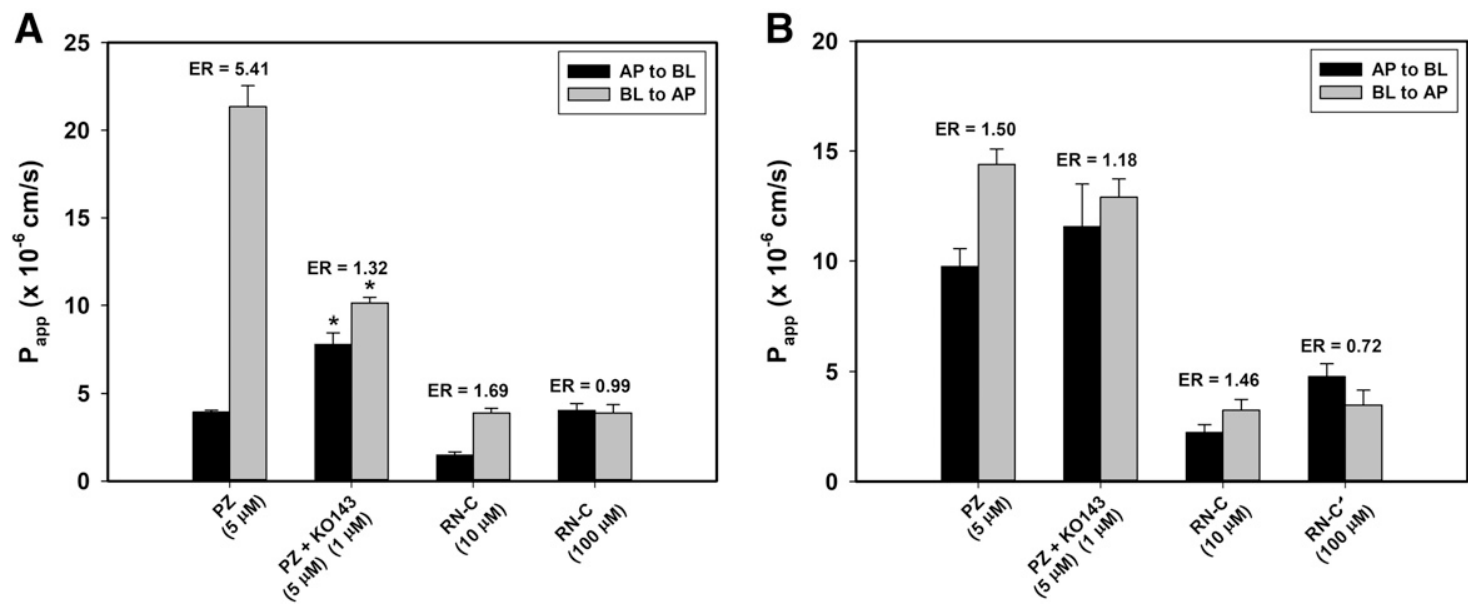

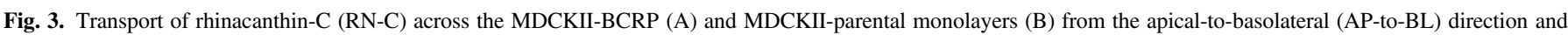


Apparent permeability coefficients (Papp) and efflux ratio (ER) represent mean \pm S.E. of four independent experiments. ${ }^{*} P<0.05$ vs .control. 
A

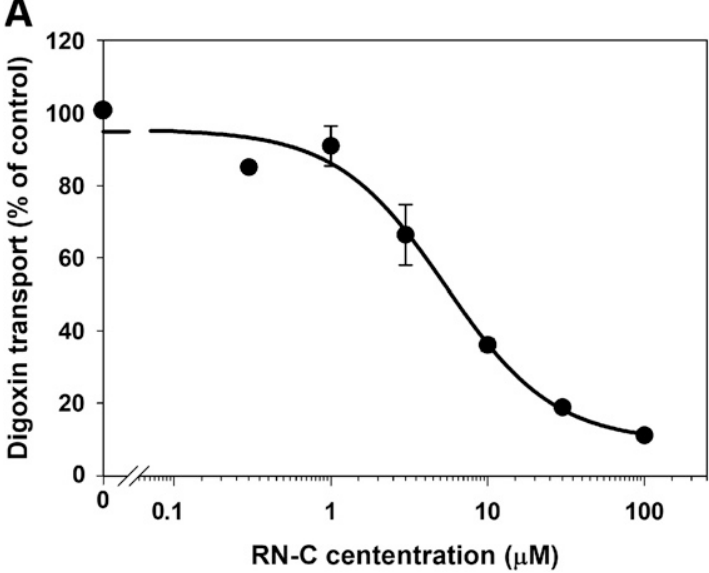

B



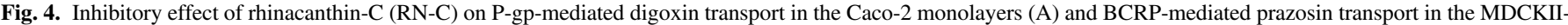

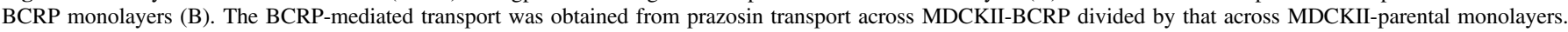
Values are expressed as percentage of vehicle control. Each value represents the mean \pm S.E. of three independent experiments.

by monitoring the uptake of probe substrate 8-FcA in the HEK293 cell line heterologously expressing the human OATP1B1 or OATP1B3. Under our conditions, the positive control inhibitor cyclosporin-A inhibited OATP1B1 and OATP1B3 with $\mathrm{IC}_{50}$ values of $0.71 \pm 0.19$ and $0.31 \pm 0.11 \mu \mathrm{M}$, respectively. In addition, rifampicin inhibited OATP1B1 and OATP1B3, with $\mathrm{IC}_{50}$ values of $1.44 \pm 0.49$ and $1.47 \pm 0.52 \mu \mathrm{M}$, respectively. Rhinacanthin-C was able to inhibit both OATP1B1-mediated and OATP1B3-mediated uptake of 8-FcA in a concentration-dependent manner (Fig. 5). This compound inhibited OATP1B1 with $\mathrm{IC}_{50}$ values of $0.70 \pm 0.12 \mu \mathrm{M}$ and inhibited OATP1B3 with $\mathrm{IC}_{50}$ values of $3.95 \pm$ $1.36 \mu \mathrm{M}$ (Table 3). Apparently, rhinacanthin-C was approximately 8 -fold more selective for OATP1B1 than for OATP1B3. At $10 \mu \mathrm{M}$, rhinacanthin-C suppressed activities of OATP1B1 by $87 \%$ and OATP1B3 by $65 \%$.

CYP Inhibition by Rhinacanthin-C. Inhibition of human CYP enzymes by rhinacanthin- $\mathrm{C}$ was investigated by measuring the metabolite formation of each selective probe substrates in HLMs (Table 2). Rhinacanthin-C inhibited the activities of the CYP2C family-namely CYP2C8, CYP2C9, and CYP2C19-in a concentration-dependent manner, with $\mathrm{IC}_{50}$ values of $4.45 \pm 0.44,1.57 \pm 0.22$, and $29.40 \pm$ $4.16 \mu \mathrm{M}$, respectively (Fig. 6B; Table 4). In contrast, rhinacanthin-C at all concentrations tested $(0.1-50 \mu \mathrm{M})$ did not inhibit the activities of CYP1A2, CYP2A6, CYP2B6, CYP2D6, CYP2E1, or CYP3A4/5 $\left(\mathrm{IC}_{50}>50 \mu \mathrm{M}\right)$ (Fig. 6, A, C, and D; Table 4). Because CYP3A4/5 is highly expressed in enterocytes, where high concentrations of rhinacanthin- $\mathrm{C}$ would be anticipated, the inhibition study of CYP3A4/ 5 by rhinacanthin- $\mathrm{C}$ was performed at an expanded concentration range (1-100 $\mu \mathrm{M})$ in HLMs. Rhinacanthin-C was able to inhibit CYP3A4/ 5-mediated transformation of midazolam and testosterone, with $\mathrm{IC}_{50}$ values of $53.00 \pm 4.22$ and $81.20 \pm 6.42 \mu \mathrm{M}$, respectively (Fig. 6D).

The Mechanism-Based CYP Inhibition by Rhinacanthin-C. The $\mathrm{IC}_{50}$ ratio was obtained from the ratio of the $\mathrm{IC}_{50}$ values of rhinacanthin$\mathrm{C}$ in the absence of NADPH and in the presence of NADPH during the 30-minute preincubation. As shown in Fig. 7, the $\mathrm{IC}_{50}$ values of rhinacanthin-C-mediated inhibition of CYP2C family were comparable in the absence and presence of NADPH in the preincubating reaction mixture, resulting in the $\mathrm{IC}_{50}$ ratio values of $1(0.61 \pm 0.07-1.12 \pm 0.34)$ (Fig. 7, A-C; Table 5). It is worth noting that rhinacanthin-C in the preincubating reaction mixture with NADPH was apparently more potent than that without NADPH in reducing CYP3A4/5-mediated metabolism of testosterone, resulting in the $\mathrm{IC}_{50}$ ratio value of $1.97 \pm$ 0.44 (Fig. 7E; Table 5). However, the effect of NADPH was not observed when midazolam was used as CYP3A4 substrate in place of testosterone. The $\mathrm{IC}_{50}$ ratio of rhinacanthin-C for CYP3A4/5 (midazolam as the substrate) was less than 1 (Fig. 7D; Table 5).

\section{Discussion}

Rhinacanthin-C is a major active constituent in $R$. nasutus, which has been commonly used in complementary therapy for various symptoms such as fever, fluid retention, hypertension, pneumonia, hepatitis, diabetes, and cancers (Siripong et al., 2006a,b; Horii et al., 2013; Shah et al., 2018). This compound is very likely to be taken concomitantly with several other drug substances, leading to herb-drug interaction issues (Horii et al., 2013; Shah et al., 2018). In the present study, we assessed the potential for rhinacanthin-C as a perpetrator in herb-drug

TABLE 3

$\mathrm{IC}_{50}$ values and $I_{\text {gut }} / \mathrm{IC}_{50}$ ratios for inhibition of drug transporters by rhinacanthin-C.

All data are expressed as mean \pm S.E. of three independent experiments.

\begin{tabular}{llcccc}
\hline Transporter & Substrate & Concentration $(\mu \mathrm{M})$ & $\mathrm{IC}_{50}(\mu \mathrm{M})$ & $\mathrm{I}_{\text {gut }} / \mathrm{IC}_{50}{ }^{a}$ & Potential for DDI $^{b}$ \\
\hline P-gp & Digoxin & 5.00 & $5.20 \pm 0.44$ & $8.00-32.06$ & Yes (intestinal) \\
BCRP & Prazosin & 5.00 & $0.83 \pm 0.09$ & $50.11-200.86$ & Yes (intestinal) \\
OATP1B1 & 8-FcA & 1.25 & $0.70 \pm 0.12$ & NA $^{c}$ & NA $^{c}$ \\
OATP1B3 & 8-FcA & 2.50 & $3.95 \pm 1.36$ & NA $^{c}$ & NA $^{c}$ \\
\hline
\end{tabular}

${ }^{a} I_{\text {gut }}$ of rhinacanthin-C $=41.59-166.71 \mu \mathrm{M}$ (Gotoh et al., 2004; Panichayupakaranant et al., 2009).

${ }^{b}$ Possible DDI risk based on $I_{\text {gut }} / \mathrm{IC}_{50}>10$, where $I_{\text {gut }}$ is the intestinal luminal concentration of the interaction drug [calculated from dose (mol) $/ 250 \mathrm{ml}$ ], as described in Zhang et al., 2008, Giacomini et al., 2010; CDER, 2017a.

${ }^{c} \mathrm{NA}$, not applicable: transporter not highly expressed in the gastrointestinal tract (Hilgendorf et al., 2007). 
A

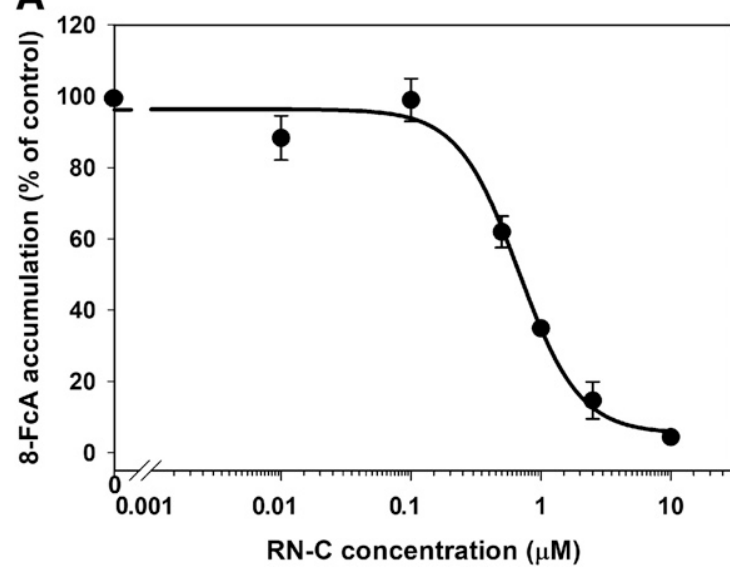

B

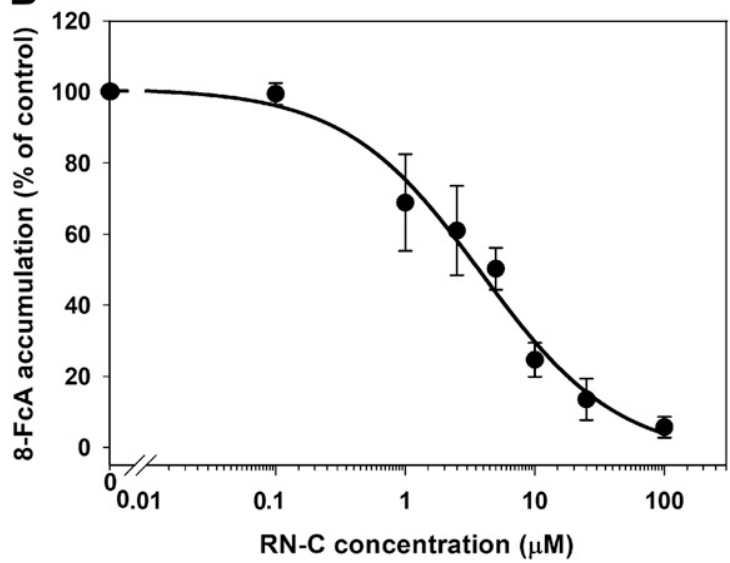

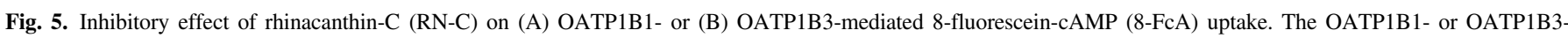

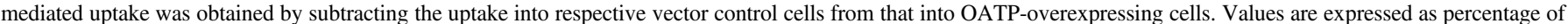
vehicle control. Each value represents the mean \pm S.E. of three independent experiments.

interaction via modulation of drug metabolizing enzymes and transporters.

To be a perpetrator in herb-drug interaction, a compound needs to be significantly absorbed through the GI epithelium (Zhou et al., 2007; Sprouse and van Breemen, 2016). Our study showed that rhinacanthin-C was not a substrate for P-gp or BCRP efflux transporters. In addition, it demonstrated moderate permeability through the model GI membrane when compared with the FDA-recommended bioavailability markers (CDER, 2017b). Thus, this compound could function as a perpetrator if it interfered with the metabolizing enzymes and transporters.

The intestinal efflux transporters play important roles in oral bioavailability and tissue distribution of their substrate drugs (Misaka et al.,




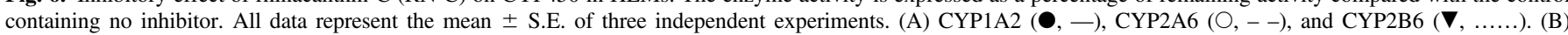


(testosterone substrate) $(\mathrm{O},--)$. 
TABLE 4

$\mathrm{IC}_{50}$ values for inhibition of cytochrome P450 enzymes by rhinacanthin-C

\begin{tabular}{|c|c|c|c|}
\hline Enzyme & Substrate & Metabolite & $\mathrm{IC}_{50}(\mu \mathrm{M})^{a}$ \\
\hline CYP1A2 & Phenacetin & Acetaminophen & $>50$ \\
\hline CYP2A6 & Coumarin & 7-Hydroxycoumarin & $>50$ \\
\hline CYP2B6 & Bupropion & Hydroxybupropion & $>50$ \\
\hline CYP2C8 & Amodiaquine & $N$-desethylamodiaquine & $4.45 \pm 0.44$ \\
\hline CYP2C9 & Tolbutamide & Hydroxytolbutamide & $1.57 \pm 0.22$ \\
\hline CYP2C19 & (S)-mephenytoin & $( \pm)-4^{\prime}$ Hydroxymephenytoin & $29.40 \pm 4.16$ \\
\hline CYP2D6 & Dextromethorphan & dextrophan & $>50$ \\
\hline CYP2E1 & Chlorzoxazone & 6-Hydroxychlorzoxazone & $>50$ \\
\hline \multirow[t]{2}{*}{ CYP3A4/5 } & Midazolam & 1'-Hydroxymidazolam & $53.00 \pm 4.22$ \\
\hline & Testosterone & $6 \beta$-Hydroxytestosterone & $81.20 \pm 6.42$ \\
\hline
\end{tabular}

${ }^{a} \mathrm{IC}_{50}$ values are expressed as the mean \pm S.E. of three independent experiments.

${ }^{b}$ Tested at the concentration range of $1-100 \mu \mathrm{M}$.

2013; Wu et al., 2016). In addition, the liver also expresses high levels of CYP enzymes and influx transporters (Wienkers and Heath, 2005; König et al., 2013). The inhibitory potential of rhinacanthin-C on these enzymes and transporters in the hepatic and other organs will result in alteration in the pharmacokinetic profile of the coadministered drug. Our results suggest that rhinacanthin- $\mathrm{C}$ is capable of selective inhibition of drug transporters and multiple CYP isoforms.

Rhinacanthin- $\mathrm{C}$ was capable of inhibiting both efflux and influx transporters including P-gp, BCRP, OATP1B1, and OATP1B3. Inhibition of intestinal P-gp or BCRP efflux transporters has been correlated to increase bioavailability and plasma concentration of their substrate drugs such as topotecan (Pgp/BCRP), paclitaxel, digoxin, indinavir (P-gp), and rosuvastatin (BCRP) (Kruijtzer et al., 2002; Hendrikx et al., 2013; Misaka et al., 2013; Elsby et al., 2016). Recently, we reported that rhinacanthin-C could interfere with $\mathrm{P}$-gp function in the Caco-2 and MCF-7 cell models (Wongwanakul et al., 2013; Chaisit et al., 2017). In this study, rhinacanthin-C displayed higher potency and selectivity for BCRP as compared with P-gp in the bidirectional transport assay, with the $\mathrm{IC}_{50}$ values of $0.83 \mu \mathrm{M}$ (BCRP) and $5.20 \mu \mathrm{M}$ (P-gp), respectively.

We further assessed its perpetrator potential to mediate in vivo intestinal efflux transporter-based interaction by calculating the $I_{\text {gut }} / \mathrm{IC}_{50}$ ratio values (Table 3) (CDER, 2017a). Given that the recommended dose of $R$. nasutus capsule (rhinacanthin-C content $\sim 0.47-1.90 \% \mathrm{w} / \mathrm{w}$ ) is 900 $\mathrm{mg}$, the intestinal amount of rhinacanthin- $\mathrm{C}$ may range approximately from 4.27 to $17.10 \mathrm{mg}$ in $250 \mathrm{ml}$ GI volume (Gotoh et al., 2004; Panichayupakaranant et al., 2009). The in vitro calculated $I_{\text {gut }} / \mathrm{IC}_{50}$ values for BCRP was $>50$, suggesting a high risk of in vivo herb-drug interaction arising from rhinacanthin-C-mediated inhibition of intestinal BCRP (CDER, 2017a). In addition, a potential in vivo drug interaction associated with rhinacanthin-C-mediated intestinal $\mathrm{P}$-gp inhibition also existed ( $I_{\text {gut }} / \mathrm{IC}_{50}$ ratios ranging from 8 to 32 ; Table 3$)$, depending on the dose of $R$. nasutus and its rhinacanthin-C content.

The inhibitory effect of rhinacanthin-C against OATP1B1 and OATP1B3 influx transporters was also evaluated. This compound demonstrated higher potency and selectivity for OATP1B1 $\left(\mathrm{IC}_{50}\right.$ $0.70 \mu \mathrm{M})$ than for OATP1B3 $\left(\mathrm{IC}_{50} 3.95 \mu \mathrm{M}\right)$. The inhibitory potency of rhinacanthin-C was quite comparable with that of the known OATP1B1 inhibitor cyclosporin A ( $\left.\mathrm{IC}_{50} 0.71 \mu \mathrm{M}\right)$. Both OATP1B1 and OATP1B3 influx transporters are highly expressed in the liver and play an important role in drug disposition (König et al., 2013). Inhibition of these two influx transporters results in an increased plasma concentration of their drug substrates such as pitavastatin, pravastatin, fexofenadine, and methotrexate (Kalliokoski and Niemi, 2009; König et al., 2013). Hence, our results support a high potential for rhinacanthin$\mathrm{C}$ to cause clinically significant drug interaction with several drugs that are substrates of multiple drug transporters such as digoxin, doxorubicin (P-gp substrate), atorvastatin, rosuvastatin, and simvastatin (BCRP and OATP substrates).

Inhibition of CYP enzyme-mediated metabolism can be attributed to as high as $70 \%$ of drug interaction issues (Han, 2011). In this study, we assessed the inhibitory potential of rhinacanthin-C on nine CYP isoforms (i.e., CYP1A2, CYP2A6, CYP2B6, CYP2C8, CYP2C9, CYP2C19, CYP2D6, CYP2E1, and CYP3A4/5) in HLMs. Our data clearly showed that rhinacanthin-C strongly inhibited several CYP2C isoforms (CYP2C8, IC ${ }_{50} 4.45 \mu \mathrm{M}$; CYP2C9, IC $501.57 \mu \mathrm{M}$; CYP2C19, $\mathrm{IC}_{50} 29.40 \mu \mathrm{M}$ ), but not CYP1A2, CYP2A6, CYP2B6, CYP2D6, and CYP2E1. Although rhinacanthin-C was previously reported to inhibit reconstituted recombinant CYP2A6 (Pouyfung et al., 2014), we did not detect its CYP2A6 inhibition in our HLM-based assay system. This disparity may be attributed to the difference in CYP functionality in the enzyme sources used (i.e., recombinant CYP vs. HLMs). Critical differences in the sensitivity to detect time-dependent CYP inactivation arising from different enzyme sources were recently reported (Di et al., 2007; Kahma et al., 2019). The inhibitory action of rhinacanthin-C against CYP2C isoforms was NADPH-independent, suggesting a nonmechanism-based inhibition. Nevertheless, we could not rule out the possibility that rhinacanthin-C might be a substrate of these CYP isoforms.

In addition, this compound demonstrated weak inhibitory effect against CYP3A4/5 with the $\mathrm{IC}_{50}$ values of $53 \mu \mathrm{M}$ (midazolam, substrate) and $81 \mu \mathrm{M}$ (testosterone, substrate). It is interesting to note that rhinacanthin-C-mediated inhibition of testosterone metabolism was NADPH dependent, suggesting mechanism-based inhibition $\left(\mathrm{IC}_{50}\right.$ ratio $=1.97$, Table 5) (Haque et al., 2017). However, this NADPH-dependent CYP3A4/5 inhibition was not observed when midazolam was used as CYP3A4/5 substrate $\left(\mathrm{IC}_{50}\right.$ ratio $<1$, Table 5) (Haque et al., 2017). These findings indicate that rhinacanthin- $\mathrm{C}$ had low risk for herb-drug interaction via CYP3A4/5.

Our results suggest that a potential risk of herb-drug interaction arising from rhinacanthin-C-mediated CYP inhibition is likely to be associated with inhibition of CYP2C9. Inhibition of CYP2C9 could affect the metabolism of its drug substrates such as $(S)$-warfarin, tolbutamide, and phenytoin (van Booven et al., 2010). Inactivation of CYP2C9 by desethylamiodarone $\left(\mathrm{IC}_{50}\right.$ $5.5 \mu \mathrm{M})$ has been reported to be a major contributor to drug interaction between $(S)$-warfarin and amiodarone, leading to an increased plasma concentration of $(S)$-warfarin and risk of hemorrhage (Heimark et al., 1992; McDonald et al., 2012). It is very likely that rhinacanthin- $\mathrm{C}$ is capable of interfering with the CYP2C9-mediated metabolism of its substrate drugs when used concurrently. 
A

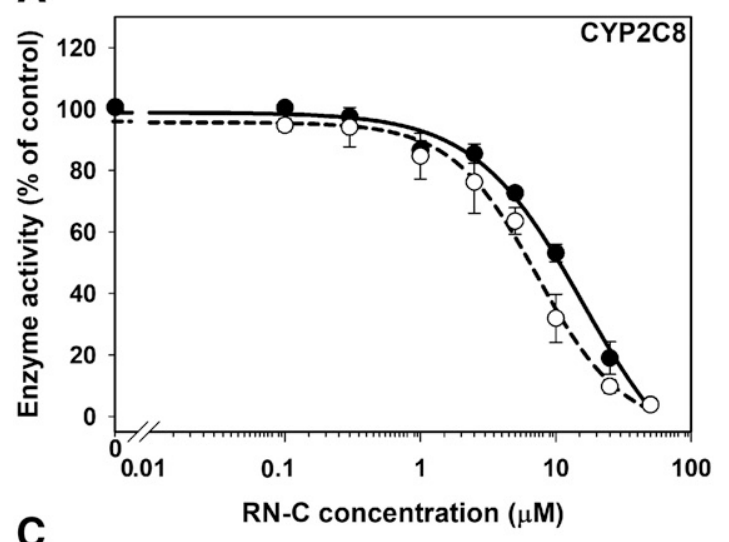

C

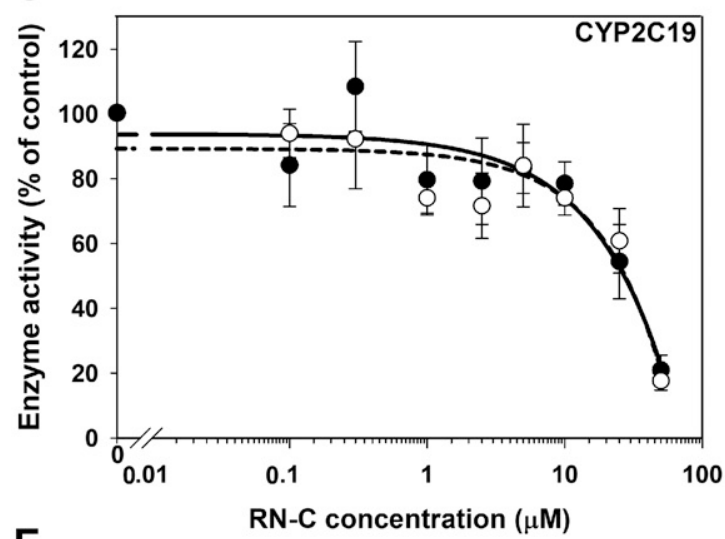

E

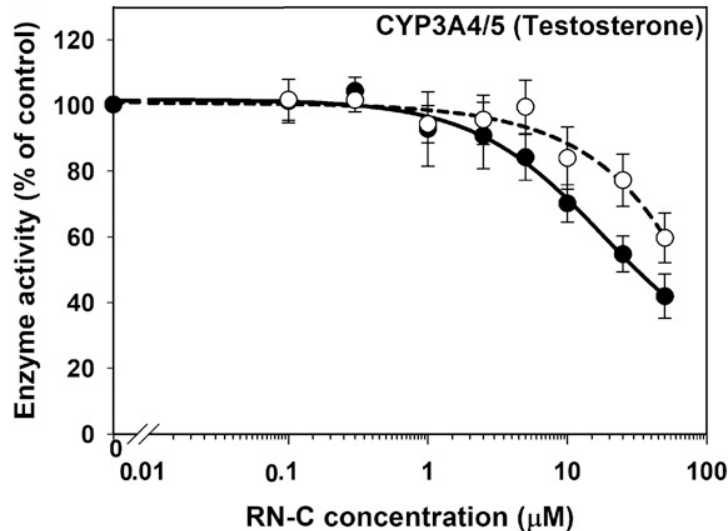

B


Fig. 7. The NADPH-dependent inhibition of rhinacanthin-C (RN-C) against (A) CYP2C8, (B) CYP2C9, (C) CYP2C19, (D) CYP3A4/5 for midazolam, and (E) CYP3A4/5 for testosterone with NADPH preincubation $(\bullet,-)$ or without NADPH preincubation $(\bigcirc,---)$ in HLMs. The enzyme activity is expressed as a percentage of remaining activity compared with the control containing no inhibitor. All data represent the mean \pm S.E. of three independent experiments.

CYP2C9 is primarily expressed in the liver and intestine (Paine et al., 2006). However, its catalytic activity and content in the intestine are known to be an order of magnitude lower than those in the liver. Though the intestinal CYP2C9 has a low contribution to first-pass metabolism of substrate drugs, this enzyme shows large interindividual variability in its activity and content (Paine et al., 2006; Xie et al., 2016). Thus, CYP enzyme inhibitors may cause drug interactions via intestinal CYP2C9 in some individuals, especially in cases of low oral bioavailability substrate drugs (Paine et al., 2006; Xie et al., 2016). For example, coadministration of fluvastatin with CYP2C9 inhibitors (e.g., ranitidine, cimetidine, and omeprazole) increased the bioavailability of fluvastatin (Scripture and Pieper, 2001). Based on the basic likelihood DDI model of CYP inhibition, the predicted ratio $(\mathrm{R})$ can be calculated from $1+\left(I_{\max , \mathrm{u}} / K_{\mathrm{i}}\right)$, where $I_{\max , \mathrm{u}}$ is the maximum unbound plasma concentration of rhinacanthin-C and $K_{\mathrm{i}}$ is the in vitro unbound inhibition constant (CDER, 2017a). The $K_{\mathrm{i}}$ value of $0.79 \mu \mathrm{M}$ was estimated from the Cheng-Prusoff equation for competitive inhibition, $K_{\mathrm{i}}=\mathrm{IC}_{50} /(1+[\mathrm{S}] /$ $K_{\mathrm{m}}$ ) (Hutzler et al., 2011). Given that the in vivo CYP inhibition is likely to occur if $\mathrm{R} \geq 1.02$ (CDER, 2017a), the maximum plasma concentration of rhinacanthin-C should be less than $0.016 \mu \mathrm{M}$ to prevent drug interaction issues with coadministered CYP2C9 substrate drugs. However, there is currently no information on plasma concentration of rhinacanthin- $\mathrm{C}$ available. Thus, the in vivo herbdrug interaction from this compound at the liver could not be predicted. On the other hand, in our study the expected concentration of rhinacanthin- $\mathrm{C}$ in the intestine was as high as 26-fold of its 
TABLE 5

$\mathrm{IC}_{50}$ values and ratio for inhibition of CYP2C8, 2C9, 2C19, and 3A4/5 with and without NADPH by rhinacanthin-C

All data represent the mean \pm S.E. of three independent experiments.

\begin{tabular}{|c|c|c|c|c|}
\hline \multirow{2}{*}{ Enzyme } & \multirow{2}{*}{ Substrate } & \multicolumn{3}{|c|}{$\mathrm{IC}_{50}(\mu \mathrm{M})$} \\
\hline & & Without NADPH Preincubation & With NADPH Preincubation & $\mathrm{IC}_{50}$ Ratio $^{a}$ \\
\hline CYP2C8 & Amodiaquine & $6.58 \pm 1.22$ & $10.68 \pm 0.74$ & $0.61 \pm 0.07$ \\
\hline CYP2C9 & Tolbutamide & $2.71 \pm 0.38$ & $3.99 \pm 0.58$ & $0.68 \pm 0.05$ \\
\hline CYP2C19 & $(S)$-mephenytoin & $25.03 \pm 5.41$ & $24.44 \pm 3.79$ & $1.12 \pm 0.34$ \\
\hline \multirow[t]{2}{*}{ CYP3A4/5 } & Midazolam & $32.70 \pm 4.30$ & $34.50 \pm 1.60$ & $0.94 \pm 0.09$ \\
\hline & Testosterone & $50.60 \pm 4.42$ & $27.20 \pm 3.26$ & $1.97 \pm 0.44$ \\
\hline
\end{tabular}

${ }^{a} \mathrm{IC}_{50}$ ratio was calculated by the $\mathrm{IC}_{50}$ value of rhinacanthin-C without NADPH preincubation divided by the $\mathrm{IC}_{50}$ value of rhinacanthin-C with NADPH preincubation.

$\mathrm{IC}_{50}$ value for CYP2C9, and the herb-drug interaction at the intestinal site could be anticipated.

Rhinacanthin-C may contribute to the therapeutic efficacy of $R$. nasutus (Sendl et al., 1996; Siripong et al., 2006a,b; Panichayupakaranant et al., 2009). However, a potential safety risk stemming from herb-drug interaction may also exist when this natural substance is coadministered with drug substrates of the CYP2C family, OATP1B1/ OATP1B3 influx transporters, and P-gp/BCRP efflux transporters. The risk of adverse events may increase when those drug substrates are in the "narrow therapeutic" drug group such as digoxin (P-gp substrate), warfarin, and phenytoin (CYP2C9 substrate) (van Booven et al., 2010; Misaka et al., 2013). In addition, several drugs can be substrates of both CYP450 enzymes and drug transporters. For example, repaglinide is a substrate of CYP2C8 and OATP1B1 (Bidstrup et al., 2003). Pitavastatin and rosuvastatin are known substrates of OATP, BCRP, and CYP2C9 (Causevic-Ramosevac and Semiz, 2013; Hu and Tomlinson, 2014).

Clinical pharmacokinetic drug interaction arising from CYP enzymes and OATP inhibition was reported in a case of combination use of gemfibrozil (CYP2C8 and OATP1B1 inhibitor) and cerivastatin (CYP2C8 and OATP1B1 substrate) (Backman et al., 2002; Shitara et al., 2004). Gemfibrozil and its metabolites increased the plasma concentration of cerivastatin via inhibiting both CYP2C8-mediated cerivastatin metabolism and OATP1B1-mediated cerivastatin hepatic uptake, leading to a high risk of rhabdomyolysis (Backman et al., 2002; Shitara et al., 2004). Because rhinacanthin-C can inhibit multiple CYP isoforms, the potential for significant pharmacokinetic herb-drug interactions must be kept in mind when it is used in conjunction with other drugs that are substrates for these enzymes. Special attention should be directed toward those that are substrates of CYP2C8, CYP2C9, and CYP2C19.

In conclusion, this in vitro study revealed that rhinacanthin-C is capable of inhibiting multiple efflux and influx drug transporters (i.e., P-gp, BCRP, OATP1B1, and OATP1B3) and CYP isoforms (i.e., CYP2C8, CYP2C9, CYP2C19, and CYP3A4/5). The safety profile associated with herb-drug interaction issues from rhinacanthin-C should not be ignored. Further studies on in vivo pharmacokinetic drug interaction should be pursued to support safe use of herbal products containing rhinacanthin- $\mathrm{C}$ in combination with other prescription drugs.

\section{Acknowledgments}

We thank Chulalongkorn University Drugs and Health Products Innovation Promotion Center for their facilities.

\section{Authorship Contributions}

Participated in research design: Dunkoksung, Vardhanabhuti, Jianmongkol. Conducted experiments: Dunkoksung.

Contributed new reagents or analytic tools: Dunkoksung, Siripong, Jianmongkol.

Performed data analysis: Dunkoksung.
Wrote or contributed to the writing of the manuscript: Dunkoksung, Vardhanabhuti, Jianmongkol.

\section{References}

Backman JT, Kyrklund C, Neuvonen M, and Neuvonen PJ (2002) Gemfibrozil greatly increases plasma concentrations of cerivastatin. Clin Pharmacol Ther 72:685-691.

Bednarczyk D (2010) Fluorescence-based assays for the assessment of drug interaction with the human transporters OATP1B1 and OATP1B3. Anal Biochem 405:50-58.

Bidstrup TB, Bjørnsdottir I, Sidelmann UG, Thomsen MS, and Hansen KT (2003) CYP2C8 and CYP3A4 are the principal enzymes involved in the human in vitro biotransformation of the insulin secretagogue repaglinide. Br J Clin Pharmacol 56:305-314.

Brantley SJ, Argikar AA, Lin YS, Nagar S, and Paine MF (2014) Herb-drug interactions: challenges and opportunities for improved predictions. Drug Metab Dispos 42:301-317.

Causevic-Ramosevac A and Semiz S (2013) Drug interactions with statins. Acta Pharm 63: 277-293.

Center for Drug Evaluation and Research (CDER) (2017a) In Vitro Metabolism- and TransporterMediated Drug-Drug Interaction Studies Guidance for Industry [Draft Guidance], U.S. Department of Health and Human Services, Food and Drug Administration, Rockville, MD. https:// www.fda.gov/downloads/Drugs/Guidances/UCM581965.pdf.

Center for Drug Evaluation and Research (CDER) (2017b) Waiver of In Vivo Bioavailability and Bioequivalence Studies for Immediate-Release Solid Oral Dosage Forms Based on a Biopharmaceutics Classification System: Guidance for Industry [Docket FDA-2015-D-1245], U.S. Department of Health and Human Services, Food and Drug Administration, Rockville, MD. https://www.fda.gov/downloads/Drugs/Guidances/ucm070246.pdf.

Chaisit T, Siripong P, and Jianmongkol S (2017) Rhinacanthin-C enhances doxorubicin cytotoxicity via inhibiting the functions of P-glycoprotein and MRP2 in breast cancer cells. Eur J Pharmacol 795:50-57.

de Ron L and Rajaraman G (2012) Assessment of the time-dependent inhibition (TDI) potential of test compounds with human liver microsomes by $\mathrm{IC}_{50}$ shift method using a nondilution approach. Curr Protoc Pharmacol Chapter 7:Unit7.14.

Di L, Kerns EH, Li SQ, and Carter GT (2007) Comparison of cytochrome P450 inhibition assays for drug discovery using human liver microsomes with LC-MS, rhCYP450 isozymes with fluorescence, and double cocktail with LC-MS. Int J Pharm 335:1-11.

Dunkoksung W, Vardhanabhuti N, and Jianmongkol S (2019) Potential P-glycoprotein-mediated herb-drug interaction of phyllanthin at the intestinal absorptive barrier. J Pharm Pharmacol 71: 213-219.

Elsby R, Martin P, Surry D, Sharma P, and Fenner K (2016) Solitary inhibition of the breast cancer resistance protein efflux transporter results in a clinically significant drug-drug interaction with rosuvastatin by causing up to a 2 -fold increase in statin exposure. Drug Metab Dispos 44: $398-408$.

Giacomini KM, Huang SM, Tweedie DJ, Benet LZ, Brouwer KL, Chu X, Dahlin A, Evers R, Fischer V, Hillgren KM, et al.; International Transporter Consortium (2010) Membrane transporters in drug development. Nat Rev Drug Discov 9:215-236.

Gotoh A, Sakaeda T, Kimura T, Shirakawa T, Wada Y, Wada A, Kimachi T, Takemoto Y, Iida A Iwakawa S, et al. (2004) Antiproliferative activity of Rhinacanthus nasutus (L.) Kurz extracts and the active moiety, Rhinacanthin C. Biol Pharm Bull 27:1070-1074.

Gurley BJ, Gardner SF, Hubbard MA, Williams DK, Gentry WB, Khan IA, and Shah A (2005) In vivo effects of goldenseal, kava kava, black cohosh, and valerian on human cytochrome P450 1A2, 2D6, 2E1, and 3A4/5 phenotypes. Clin Pharmacol Ther 77:415-426.

Han HK (2011) Role of transporters in drug interactions. Arch Pharm Res 34:1865-1877.

Haque AKMM, Leong KH, Lo YL, Awang K, and Nagoor NH (2017) In vitro inhibitory mechanisms and molecular docking of $1^{\prime}-S-1^{\prime}$-acetoxychavicol acetate on human cytochrome P450 enzymes. Phytomedicine 31:1-9.

Heimark LD, Wienkers L, Kunze K, Gibaldi M, Eddy AC, Trager WF, O'Reilly RA, and Goulart DA (1992) The mechanism of the interaction between amiodarone and warfarin in humans. Clin Pharmacol Ther 51:398-407.

Hendrikx JJ, Lagas JS, Rosing H, Schellens JH, Beijnen JH, and Schinkel AH (2013) P-glycoprotein and cytochrome P450 3A act together in restricting the oral bioavailability of paclitaxel. Int J Cancer 132:2439-2447.

Hilgendorf C, Ahlin G, Seithel A, Artursson P, Ungell AL, and Karlsson J (2007) Expression of thirty-six drug transporter genes in human intestine, liver, kidney, and organotypic cell lines. Drug Metab Dispos 35:1333-1340.

Horii H, Suzuki R, Sakagami H, Tomomura M, Tomomura A, and Shirataki Y (2013) New biological activities of Rhinacanthins from the root of Rhinacanthus nasutus. Anticancer Res 33:453-459.

Hu M and Tomlinson B (2014) Evaluation of the pharmacokinetics and drug interactions of the two recently developed statins, rosuvastatin and pitavastatin. Expert Opin Drug Metab Toxicol 10: $51-65$. 
Hubatsch I, Ragnarsson EG, and Artursson P (2007) Determination of drug permeability and prediction of drug absorption in Caco-2 monolayers. Nat Protoc 2:2111-2119.

Hutzler JM, Cook J, and Fleishaker JC (2011) Drug-drug interactions: designing development programs and appropriate product labeling, in Pharmacokinetics in Drug Development (Bonate PL and Howard DR eds) pp 21-56, Springer, New York.

Kahma H, Filppula AM, Launiainen T, Viinamäki J, Neuvonen M, Evangelista EA, Totah RA, and Backman JT (2019) Critical differences between enzyme sources in sensitivity to detect time-dependent inactivation of CYP2C8. Drug Metab Dispos 47:436-443.

Kalliokoski A and Niemi M (2009) Impact of OATP transporters on pharmacokinetics. Br J Pharmacol 158:693-705.

König J, Glaeser H, Keiser M, Mandery K, Klotz U, and Fromm MF (2011) Role of organic aniontransporting polypeptides for cellular mesalazine (5-aminosalicylic acid) uptake. Drug Metab Dispos 39:1097-1102

König J, Klatt S, Dilger K, and Fromm MF (2012) Characterization of ursodeoxycholic and norursodeoxycholic acid as substrates of the hepatic uptake transporter OATP1B1, OATP1B3, OATP2B1 and NTCP. Basic Clin Pharmacol Toxicol 111 $81-86$.

König J, Müller F, and Fromm MF (2013) Transporters and drug-drug interactions: important determinants of drug disposition and effects. Pharmacol Rev 65:944-966.

Kruijtzer CM, Beijnen JH, Rosing H, ten Bokkel Huinink WW, Schot M, Jewell RC, Paul EM, and Schellens JH (2002) Increased oral bioavailability of topotecan in combination with the breast cancer resistance protein and P-glycoprotein inhibitor GF120918. J Clin Oncol 20: 2943-2950.

Li G, Huang K, Nikolic D, and van Breemen RB (2015) High-throughput cytochrome P450 cocktail inhibition assay for assessing drug-drug and drug-botanical interactions. Drug Metab Dispos 43:1670-1678.

McDonald MG, Au NT, Wittkowsky AK, and Rettie AE (2012) Warfarin-amiodarone drug-drug interactions: determination of $[\mathrm{I}](\mathrm{u}) / \mathrm{K}(\mathrm{I}, \mathrm{u})$ for amiodarone and its plasma metabolites. Clin Pharmacol Ther 91:709-717.

Miners JO and Birkett DJ (1998) Cytochrome P4502C9: an enzyme of major importance in human drug metabolism. Br J Clin Pharmacol 45:525-538

Misaka S, Müller F, and Fromm MF (2013) Clinical relevance of drug efflux pumps in the gut Curr Opin Pharmacol 13:847-852.

Mizuno N, Niwa T, Yotsumoto Y, and Sugiyama Y (2003) Impact of drug transporter studies on drug discovery and development. Pharmacol Rev 55:425-461.

Obach RS (2008) Metabolizing enzymes and drug-drug interactions in drug discovery and development, in Drug-Drug Interactions in Pharmaceutical Development (Li AP ed) pp 75-93, John Wiley \& Sons, Inc., Hoboken, NJ.

Oga EF, Sekine S, Shitara Y, and Horie T (2016) Pharmacokinetic herb-drug interactions: insight into mechanisms and consequences. Eur J Drug Metab Pharmacokinet 41 93-108

Paine MF, Hart HL, Ludington SS, Haining RL, Rettie AE, and Zeldin DC (2006) The human intestinal cytochrome P450 "pie". Drug Metab Dispos 34:880-886.

Panichayupakaranant P, Charoonratana T, and Sirikatitham A (2009) RP-HPLC analysis of rhinacanthins in Rhinacanthus nasutus: validation and application for the preparation of rhinacanthin high-yielding extract. $J$ Chromatogr Sci 47:705-708.

Poller B, Wagenaar E, Tang SC, and Schinkel AH (2011) Double-transduced MDCKI cells to study human P-glycoprotein $(\mathrm{ABCB} 1)$ and breast cancer resistance protein (ABCG2) interplay in drug transport across the blood-brain barrier. Mol Pharm 8 $571-582$.
Pouyfung P, Prasopthum A, Sarapusit S, Srisook E, and Rongnoparut P (2014) Mechanism-based inactivation of cytochrome P450 2A6 and 2A13 by Rhinacanthus nasutus constituents. Drug Metab Pharmacokinet 29:75-82.

Scripture CD and Pieper JA (2001) Clinical pharmacokinetics of fluvastatin. Clin Pharmacokinet 40:263-281.

Seithel A, Eberl S, Singer K, Auge D, Heinkele G, Wolf NB, Dörje F, Fromm MF, and König J (2007) The influence of macrolide antibiotics on the uptake of organic anions and drugs mediated by OATP1B1 and OATP1B3. Drug Metab Dispos 35:779-786.

Sendl A, Chen JL, Jolad SD, Stoddart C, Rozhon E, Kernan M, Nanakorn W, and Balick M (1996) Two new naphthoquinones with antiviral activity from Rhinacanthus nasutus. J Nat Prod 59: 808-811.

Shah MA, Keach JE, and Panichayupakaranant P (2018) Antidiabetic naphthoquinones and their plant resources in Thailand. Chem Pharm Bull (Tokyo) 66:483-492.

Shitara Y, Hirano M, Sato H, and Sugiyama Y (2004) Gemfibrozil and its glucuronide inhibit the organic anion transporting polypeptide 2 (OATP2/OATP1B1:SLC21A6)-mediated hepatic uptake and CYP2C8-mediated metabolism of cerivastatin: analysis of the mechanism of the clinically relevant drug-drug interaction between cerivastatin and gemfibrozil. J Pharmacol Exp Ther 311:228-236.

Siripong P, Kanokmedakul K, Piyaviriyagul S, Yahuafal J, Chanpai R, Ruchirawat S, and Oku N (2006b) Antiproliferative naphthoquinone esters from Rhinacanthus nasutus Kurz. roots on various cancer cells. $J$ Trad Med 23:166-172.

Siripong P, Yahuafai J, Shimizu K, Ichikawa K, Yonezawa S, Asai T, Kanokmedakul K, Ruchirawat S, and Oku N (2006a) Induction of apoptosis in tumor cells by three naphthoquinone esters isolated from Thai medicinal plant: Rhinacanthus nasutus KURZ. Biol Pharm Bull 29: 2070-2076.

Sprouse AA and van Breemen RB (2016) Pharmacokinetic interactions between drugs and botanical dietary supplements. Drug Metab Dispos 44:162-171.

Van Booven D, Marsh S, McLeod H, Carrillo MW, Sangkuhl K, Klein TE, and Altman RB (2010) Cytochrome P450 2C9-CYP2C9. Pharmacogenet Genomics 20:277-281.

Wienkers LC and Heath TG (2005) Predicting in vivo drug interactions from in vitro drug discovery data. Nat Rev Drug Discov 4:825-833.

Wongwanakul R, Vardhanabhuti N, Siripong P, and Jianmongkol S (2013) Effects of rhinacanthin$\mathrm{C}$ on function and expression of drug efflux transporters in Caco-2 cells. Fitoterapia 89:80-85.

Wu X, Ma J, Ye Y, and Lin G (2016) Transporter modulation by Chinese herbal medicines and it mediated pharmacokinetic herb-drug interactions. J Chromatogr B Analyt Technol Biomed Life Sci 1026:236-253.

Xie F, Ding X, and Zhang QY (2016) An update on the role of intestinal cytochrome P450 enzymes in drug disposition. Acta Pharm Sin B 6:374-383.

Zhang L, Zhang YD, Strong JM, Reynolds KS, and Huang SM (2008) A regulatory viewpoint on transporter-based drug interactions. Xenobiotica 38:709-724.

Zhou SF, Zhou ZW, Li CG, Chen X, Yu X, Xue CC, and Herington A (2007) Identification of drugs that interact with herbs in drug development. Drug Discov Today 12:664-673.

Address correspondence to: Dr. Suree Jianmongkol, Department of Pharmacology and Physiology, Faculty of Pharmaceutical Sciences, Chulalongkorn University, Payathai Rd., Pathumwan, Bangkok 10330, Thailand. E-mail: suree.j@ pharm.chula.ac.th 